\title{
Fermi Gamma-ray Burst Monitor Capabilities for multi-messenger time-domain astronomy
}

\author{
Valerie Connaughton ${ }^{* \dagger}$ \\ Universities Space Research Association \\ E-mail: valerie@nasa.gov
}

Owing to its wide sky coverage and broad energy range, the Fermi Gamma-ray Burst Monitor $(\mathrm{GBM})$ is an excellent observer of the transient hard X-ray sky. GBM detects about 240 triggered Gamma-Ray Bursts (GRBs) per year, including 45 short GRBs, giving GBM an excellent opportunity to observe the electromagnetic counterpart to any gravitational wave candidate resulting from the merger of compact binary members. The same characteristics make GBM an ideal partner for neutrino searches from bright GRBs, and for the elusive Very-High Energy (VHE) counterparts to GRBs. With the beginning of operations of the High Altitude Water Cherenkov (HAWC) experiment, the imminent deployment of the next-generation gravitational-wave detectors (Advanced LIGO/Virgo), and the Cherenkov Telescope Array (CTA) possibly coming online within the lifetime of the Fermi Gamma-ray Space Telescope, the prospects for breakthrough observations are good.

The 34th International Cosmic Ray Conference,

30 July- 6 August, 2015

The Hague, The Netherlands

* Speaker.

${ }^{\dagger}$ for the Fermi GBM team 


\section{Introduction}

The Gamma-ray Burst Monitor (GBM) on-board the Fermi Gamma-ray Space Telescope covers the entire unocculted sky using 12 thin $(1.27 \mathrm{~cm})$ Sodium Iodide $(\mathrm{NaI})$ detectors with different orientations placed around the spacecraft and covering an energy range from $8 \mathrm{keV}$ to $1 \mathrm{MeV}$, and two bismuth germanate (BGO) scintillators placed on opposite sides of the spacecraft with energy coverage from $200 \mathrm{keV}$ to $40 \mathrm{MeV}$ [Meegan et al. 2009]. In seven years of operation, Fermi has opened a new window to the world of Gamma-Ray Burst (GRB) spectroscopy. Observations by the GBM between $8 \mathrm{keV}$ and $40 \mathrm{MeV}$ and the Large Area Telescope (LAT, [Atwood et al. 2009]) from tens $\mathrm{MeV}$ to tens of $\mathrm{GeV}$ have provided a high-energy view over an unprecedentedly long energy baseline. Popular models for GRB emission mechanisms have been challenged by the data, with observations suggesting distinct high-energy components to GRB emission that persist long after the lower-energy emission is detectable [Ackermann et al. 2013].

Follow-up observations of LAT-detected GRBs have revealed the distance to 22 GRBs. Typically the Swift X-Ray Telescope (XRT) observes the LAT error circle in one or a small number of pointings beginning from 11 to 48 hours after the GBM trigger, after ground-processing of the LAT data. This is followed by optical observations of any fading XRT afterglow candidates. In 15 LAT-detected GRBs, the latency was much smaller because the GRB also triggered the Swift BAT, and in a few LAT-detected GRBs the LAT position was accurate enough to permit optical follow-up without the intermediate stage of the Swift XRT pointing. Until 2013, the localization uncertainties associated with GBM GRBs were too large and ill-characterized to allow effective follow-up observations with most telescopes. Characterization of the GBM localization systematic uncertainties and the development and distribution of probability maps that convolve these systematic effects with the statistical uncertainty [Connaughton et al. 2015] have led to successful afterglow detections of GBM-localized GRBs by the intermediate Palomar Transient Factory (iPTF) [Singer et al. 2015], by the MASTER telescope [Gorbovskoy et al. 2014], and by the Nanshan telescope [Xu et al. 2013], and, occasionally, subsequent redshift measurements, supernova detections, and radio detections.

\section{GBM Localization of GRBs}

Source localization is achieved using the relative rates recorded in the $12 \mathrm{NaI}$ detectors to estimate the most likely arrival direction given the angular and spectral response of the detectors. In addition to background rates which average above 1000 counts/sec over the Fermi orbit and vary by a factor of about 2 depending on geomagnetic latitude, the recorded rates from the source of gamma rays contain three elements: direct flux from the source, flux scattered in the spacecraft, and flux scattered from the atmosphere. The contributions from each of these components depend on the observing geometry as well as the spectrum of gamma rays from the source. We construct our response to a particular GRB by adding two model terms, the direct response which depends only on the source-spacecraft geometry, and the atmospheric response which depends on the sourcespacecraft-Earth geometry. We compare the observed rates to the expected rates from a GRB coming from each position on the $1^{\circ}$ resolution sky grid with one of three typical input GRB spectra - soft, medium and hard - selecting the position and spectral shape that minimize $\chi^{2}$. Using 

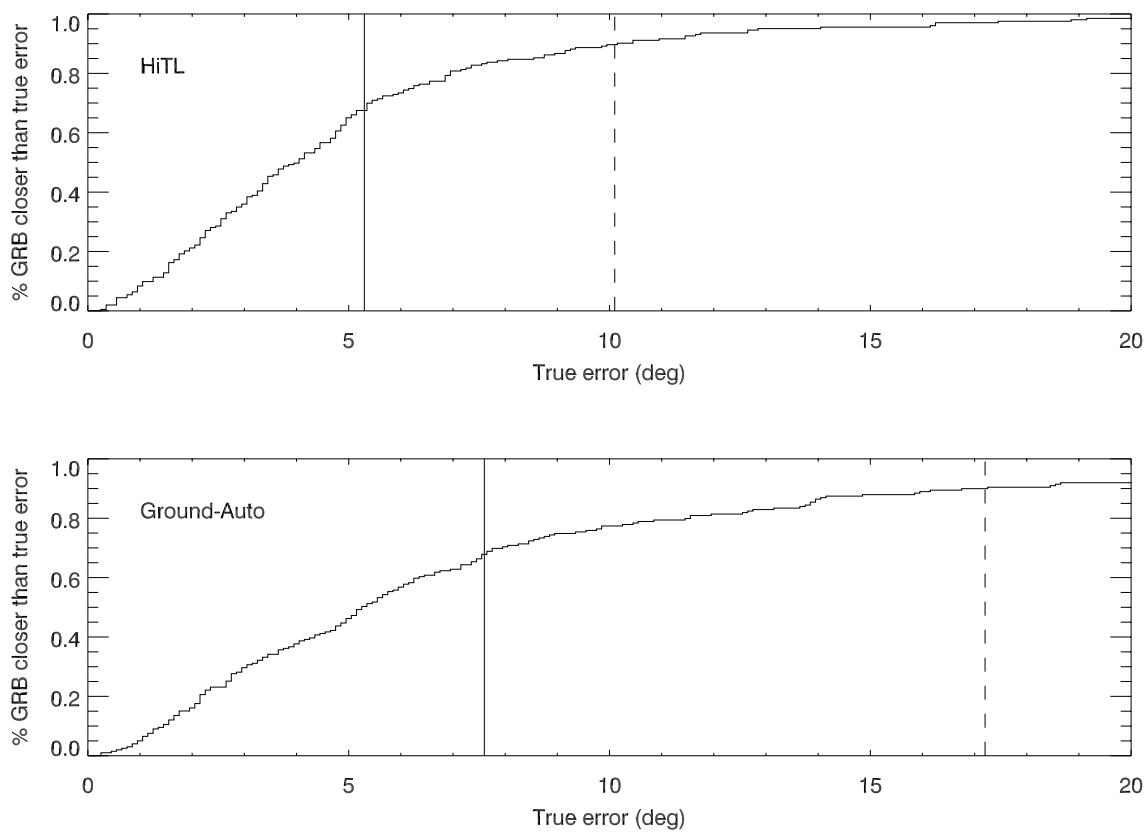

Figure 1: The histograms show the fraction of GBM localizations lying within a given offset (degrees) from the true positions for HitL (top) and Ground-automated (bottom) positions. The solid vertical lines indicate a $68 \%$ containment radius of $5.3^{\circ}\left[7.6^{\circ}\right]$, the dashed vertical lines the $90 \%$ radius of $10.1^{\circ}\left[17.2^{\circ}\right]$. From [Connaughton et al. 2015]

a sample of 200 reference GRB positions from instruments with good localizing capabilities such as the Swift BAT, Fermi LAT, INTEGRAL, MAXI, SuperAGILE, and the InterPlanetary Network (IPN), we estimate that in addition to the statistical uncertainty associated with this process, there is a $3^{\circ}$ systematic uncertainty $(1 \sigma)$ that applies to most GRBs, with about $10 \%$ of GBM-detected GRBs having a much larger systematic effect extending beyond $10^{\circ}$ [Connaughton et al. 2015].

Because of on-board computing limitations, the locations produced by the Flight Software (FSW) use a coarser $5^{\circ}$ grid, a single spectral shape, and assume the Earth is directly beneath the spacecraft. The ground automated (GA) locations and the final human-in-the-loop (HiTL) locations use the full-resolution grid and Earth-Fermi geometry, but the GA location sees only a snippet of source and background data provided by the FSW, whereas the HiTL location requires a longer data downlink and interaction by the burst advocate on duty to select both a good background and an optimized source interval. All three location types are distributed as notices to the GRB Coordinates Network (GCN). Both the FSW and GA notices arrive within seconds of data receipt, or about $30 \mathrm{~s}$ following the trigger, and the HiTL notice can arrive as soon as 20-30 minutes up to several hours after the trigger. A HiTL notice is not sent if another instrument has detected the GRB and provided a more precise location, but the FSW and GA notices appear automatically. Figure 1 shows how GBM localizations compare to known locations for 200 GRBs that were accurately localized by other instruments.

The GA localizations have recently been improved through the implementation of new algorithms to include more data and reduce the statistical uncertainty on the localization. In the coming 
months, we expect to improve this further, eventually replacing the HiTL notice with an equally good localization that will be distributed 10 minutes after the trigger time.

\section{Who should follow up GBM-detected GRBs?}

\subsection{TeV Community}

After 20 years of looking for Very High Energy (VHE) photons from GRBs, beginning in the BATSE era with the Whipple 10-m telescope [Connaughton et al. 1997], the field is still without a VHE GRB counterpart or, until recently, a meaningful upper limit. A photon with energy $E_{1}$ will interact with a photon of energy $E_{2}$ to produce an electron-positron pair if $E_{1} E_{2} *(1-\cos \theta)>$ $2\left(m_{e} c^{2}\right)^{2}$, where $\theta$ is the angle between the two photons, $m_{e}$ the rest-mass of an electron and $c$ the speed of light. This results in the attenuation of gamma-ray signals above a few tens $\mathrm{GeV}$ owing to the presence of intervening Extragalactic Background Light (EBL) photons, and limits the visible horizon so that the higher the energy of a photon, the closer the source must be in order to detect it, with the exact horizon depending on the model assumed for EBL as a function of energy and redshift. Observations of photons above $10 \mathrm{GeV}$ from GRB $080916 \mathrm{C}$ by the LAT from a redshift of 4.4, without noticeable attenuation, excluded some proposed EBL models [Abdo et al. 2009] but the remaining viable models predict that detections above a few hundred $\mathrm{GeV}$ will be possible only within redshift unity. VHE observations of GRBs are also difficult because the duty cycle of the Imaging Atmospheric Cherenkov Telescope (IACT) is low (10-13\%) owing to the need for dark, moonless nights, and the slew time to reach a GRB is typically tens of seconds to over a minute. With an energy threshold of $400 \mathrm{GeV}$, the pioneering IACT, the Whipple 10-m, had a very limited source horizon owing to EBL absorption. The lower energy threshold $(100 \mathrm{GeV})$ of the currentgeneration IACT arrays such as VERITAS means that more GRBs lie within the detectable VHE horizon compared to the Whipple era. The most recent results from VERITAS [Acciari et al. 2011] show that VHE observations have occurred within the duration of the prompt emission, but not for GRBs known to be within the VERITAS EBL horizon. The next-generation IACT, the Cherenkov Telescope Array (CTA) will be much more sensitive, with three telescope types including the Large Size Telescope (LST) which will have an energy threshold of tens GeV, bringing all but the most distant GRBs within the detection horizon of CTA. The field-of-view of the LST will be $3.5-4^{\circ}$ so that a tiling strategy for following GBM GRBs will be adopted. Estimates of the annual detection rate of GBM GRBs by CTA, taking into account this tiling necessity, the average slew rate of the telescope, and the average spectral and duration properties and distance distribution of GBMdetected GRBs, predict 0.6 - 1.6 GRBs per year for the prompt GRB emission, comparable to the expected overlap with the Swift-detected GRB sample [Gilmore et al. 2013]. The detection of temporally extended emission is common in LAT-detected GRBs so that the need to slew to the burst position will not be a limitation for detecting this emission and the number of GRBs detected may be as high as a few per year if this temporally extended emission is common in the general GRB population. High-energy emission from the exceptionally bright GRB 130427A was detected $77 \mathrm{ks}$ after the GBM trigger time [Ackermann et al. 2014]. VERITAS observations, the night after the GBM trigger, did not reveal VHE emission and allowed constraints to be placed on some of the models that explain the extended emission [Aliu et al. 2014]. 
With broad sky coverage and high duty cycle, the High Altitude Water Cherenkov (HAWC) is sensitive to bright nearby GRBs without slewing, but with lower sensitivity than CTA. HAWC is expected to detect about 1 short GRB per year [Taboada \& Gilmore 2014].

\subsection{Neutrino experiments}

Neutrinos are expected through the delta resonance from $\mathrm{p} \gamma$ interactions in the fireball, and subsequent decay of the delta resonance pion into leptons and neutrinos. For bright GRBs with Lorentz factors of around 100, this flux should be detectable with IceCube [Dermer \& Atoyan 2006]. A lack of neutrino detection constrains (i) how much energy is in protons relative to electrons in the fireball or (ii) how low the Bulk Lorentz factor of the fireball can be. The lack of neutrino detection by IceCube from a set of over 200 GRBs (a mixture of Fermi and Swift GRBs) [Abbasi et al. 2012] constrains the parameter space in at least one of these directions: either the fireball has such a high bulk Lorentz factor (greater than 400) that the true energy of the protons at the source is too low for the $\mathrm{p} \gamma$ interaction, or the amount of energy in protons is much less than $1 / 10$ the energy in electrons. For an individual GRB, the detectability of its neutrino signal depends on (i) the observed gamma-ray fluence of the GRB - the neutrino flux scales with the gamma-ray flux (ii) the Lorentz factor of the GRB - lower is better. Given that GBM is a prolific detector of bright GRBs, it is an ideal partner for neutrino searches from individual bursts in the IC59-or-beyond configurations of IceCube.

\subsection{Next-generation Gravitational Wave experiments}

Neutron Star - Neutron Star (NS-NS) and Neutron Star - Black Hole (NS-BH) mergers are believed to be likely sources of detectable gravitational waves. They are also the preferred model for short GRBs (SGRB). Searches for GW signals from 154 GRBs using past science runs from LIGO and VIRGO were not fruitful [Abadie et al. 2012], but LIGO was sensitive to SGRBs only within $33 \mathrm{Mpc}$ from NS-NS, $70 \mathrm{Mpc}$ from NS-BH systems, closer than any SGRBs with measured redshift. The expected signal from long GRBs is uncertain. The Advanced configurations of both LIGO and VIRGO will have horizons of $\mathrm{z}=0.11(445 \mathrm{Mpc})$ and $0.22(927 \mathrm{Mpc})$ for NS-NS and NSBH mergers, respectively [Abadie et al. 2010]. Estimates of annual SGRB detection within these horizons are shown in Figure 2. The SGRBs with known redshift in the Swift-detected population are assumed to form part of the same population as those with unknown redshift so that the number of SGRBs detected by Swift per year, $N_{\text {Swift }}$, as a function of redshift is presented as the number of SGRBs with measured redshift in each bin divided by the fraction of SGRBs with known redshift. The same assumption about the underlying redshift distribution of GBM-detected SGRBs yields the number of SGRBs per year seen by GBM, $N_{G B M}$, as a function of redshift. This assumption is justified by Burns et al. (in preparation), who show that the Swift BAT and Fermi GBM are sensitive to the same population of SGRB. Given that $N_{G B M} / N_{\text {Swift }}=4.5$ we show the expected SGRB detection for GBM as a function of redshift by multiplying the Swift SGRB distribution by this factor. The number of short GRBs detected by GBM will increase with the upcoming implementation of a ground search that increases the sensitivity of GBM to weaker SGRB, with a possible doubling of SGRBs suggested by extrapolation of the number-intensity distribution to the detectable weaker SGRBs. ALIGO localization of detected GW events can produce large non-contiguous sky regions covering up to 100 sq degrees, so that in addition to providing the electromagnetic counterpart to 


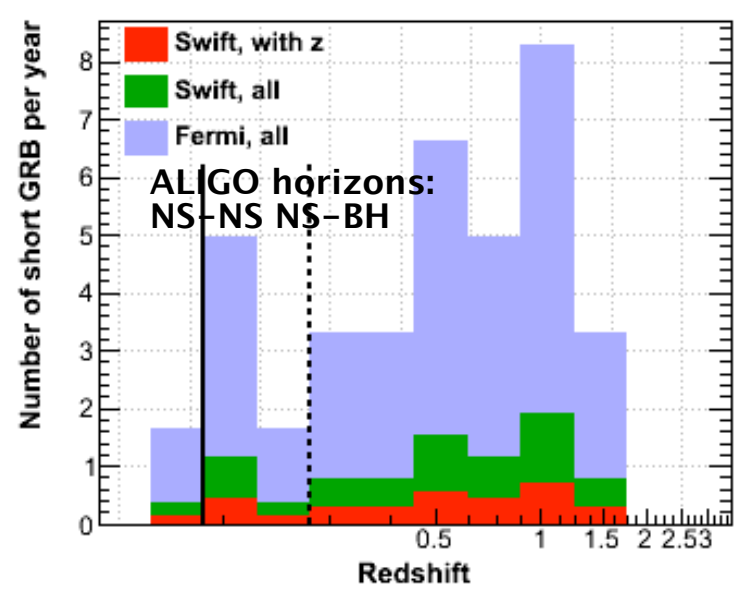

Figure 2: SGRB rate as a function of redshift for Fermi and Swift. ALIGO horizons for NS-NS and NS-BH mergers are shown as black vertical lines.

a GW candidate, the moderate localization capability (10s sq degrees) of GBM is useful in eliminating some of these regions for follow-up by X-ray or optical instruments. In the absence of a convincing GW event, GBM can also provide useful seed sky positions for sub-threshold offline ALIGO searches.

\section{The future}

Even with no improvement in localization capabilities over the Fermi mission, GBM is an ideal partner for neutrino and GW searches, particularly in the era of ALIGO/VIRGO and the deployment of new IceCube configurations with more strings. A modest improvement would really help the case for CTA, removing the need to tile the error box, and thus increasing the joint GBM-CTA GRB detection rate from a few tenths to 1 per year. Efforts to improve GBM GRB localization include: (i) characterization of systematic errors to either identify GRBs with low systematic uncertainties or refine the localization based on GRB or geometrical properties that might lead to a large but measurable systematic effect (ii) moving to a real-time GA location that uses more data and is not limited by statistics (iii) improving our detector response database by identifying its deficiencies through our study of localization errors and (iv) reducing the statistical uncertainty of GBM locations through finer grid resolution,

\section{References}

[Abadie et al. 2010] Abadie, J., Abbott, B. P., Abbott, R., et al. 2010, Class. Quant. Grav. 27:173001

[Abadie et al. 2012] Abadie, J., Abbott, B. P., Abbott, R., et al. 2012, ApJ 760, 12

[Abbasi et al. 2012] Abbasi, R., Abdou, Y., Abu-Zayyad, T., et al. 2012, Nature 484, 351

[Abdo et al. 2009] Abdo, A., Ackermann, M., Arimoto, M., et al. 2009, Science 323, 1688

[Acciari et al. 2011] Acciari, V., Aliu, E., Arlen, T., et al. 2011, ApJ 743, 62A

[Ackermann et al. 2013] Ackermann, M., Ajello, M., Asano, K. et al. 2013, ApJS 209, 11 
[Ackermann et al. 2014] Ackermann, M., Ajello, M., Asano, K. et al. 2014, Science 343, 42 Acciari, V., Aliu, E., Arlen, T., et al. 2011, ApJ 743, 62A

[Atwood et al. 2009] Atwood, W. B., Abdo, A. A., Ackermann, M., et al. 2009, ApJ 697, 1071

[Aliu et al. 2014] Aliu, E., Aune, T., Barnacka, A. et al. 2014, ApJL 795, 3

[Connaughton et al. 1997] Connaughton, V., Akerlof, C. W., Barthelmy, S., et al. 1997, ApJ 479, 859

[Connaughton et al. 2015] Connaughton, V., Briggs, M. S., Goldstein, A. et al. 2015, ApJS 216, 32

[Dermer \& Atoyan 2006] Dermer, C. D., \& Atoyan, A. 2006, New J. Phys. 8, 122

[Gilmore et al. 2013] Gilmore, R., Bouvier, A., Connaughton, V., et al. 2013, Exp.Astron. 35, 413

[Gorbovskoy et al. 2014] Gorbovskoy, E. et al 2014, GCN Circular 16653

[Greiner et al. 2009] Greiner, J., Clemens, C., Kruehler, T., et al. 2009, A\&A 498, 89

[Meegan et al. 2009] Meegan, C., Lichti, G., Bhat, P. N., et al. 2009, ApJ 702, 791

[Singer et al. 2015] Singer, S. P., Kasliwal, M. M., Cenko, S. B. et al. 2015, ApJ 806, 52

[Taboada \& Gilmore 2014] Taboada, I. \& Gilmore, R. 2014, NIM Phys.Res.A 742, 276

[Xu et al. 2013] Xu, D. et al 2013, GCN Circular 15641 